in handling the thoracoscopy equipment and IPC insertion (figure 1). $100 \%$ of consultants agreed that the course was mapped to the respiratory curriculum requirements and that the content was appropriate for ST5 trainees. 100\% of consultant faculty also agreed that the course structure was adequate; the lectures were delivered at an appropriate level and were happy for the course to be run on a yearly basis. Candidate feedback was positive and indicated that the course would be a useful addition to the training programme.

Conclusion With medical advancements and expansion of Lung Cancer Services nationwide, respiratory doctors are increasingly needed to master MT and IPC insertion skills. Intra-deanery training should be provided for trainees to ensure sustainability of services.

\section{P74 EXPERIENCES OF A SIMULATED PLEURAL BIOPSY TRAINING COURSE FOR RESPIRATORY REGISTRARS IN A HIGH TUBERCULOSIS INCIDENCE REGION OF THE UK}

${ }^{1}$ WM Ricketts, ${ }^{1} \mathrm{M}$ Farrugia, ${ }^{2} \mathrm{~J}$ Goldring, ${ }^{1} \mathrm{~T}$ O'Shaughnessy, ${ }^{3} \mathrm{M}$ Sheaff, ${ }^{4} \mathrm{DT}$ McLeod; ${ }^{1}$ Newham University Hospital, Barts Health NHS Trust, London, UK; ${ }^{2}$ Royal Free London NHS Foundation Trust, London, UK; ${ }^{3}$ Royal London Hospital, Barts Health NHS Trust, London, UK; ${ }^{4}$ Sandwell and Birmingham NHS Trust, Birmingham, UK

\subsection{6/thoraxjnl-2013-204457.224}

Introduction and Objectives Last year we presented data highlighting the on-going role for Abrams pleural biopsy in areas with high tuberculosis incidence (1). Feedback reiterated ongoing need, but highlighted concerns regarding the attainment of trainee competence at this infrequently performed procedure. We organised and evaluated a half day training course using a well validated porcine-resin model at a regional registrar training day (2).

Methods All attendees (18) underwent a practical training session on pleural biopsy using Abrams and Tru-Cut biopsy needles. Feedback forms were completed and perceived success documented, all samples obtained underwent histological assessment by a specialist respiratory Consultant Histopathologist.

Results Previous experience was limited (median 0.5 prior biopsies performed, range 0-50). On Likert scales (range 1-5) mean confidence improved (1.86, SD 1.21 to 3.83 , SD 0.51 ; p < 0.0001 ) and the session was deemed useful (mean score 4.5, SD $0.4)$ with $13 / 14(92.9 \%)$ trainees who answered stating the session would change their practice. A mean of 4.56 samples (SD 1.42) were obtained per trainee. Sixty-nine of seventy-six samples $(90.8 \%)$ were perceived to have been successful by the operator, microscopic evidence of mesothelial lined pleura was obtained in nine samples $(11.8 \%)$ with connective tissue suggestive of possible pleura obtained in a further 25 (33\%) $(\kappa=0.013$; poor correlation); real time observation by a training partner suggested a $91.9 \%$ success rate $(\kappa=0.584$; moderate correlation). There was no increase in accuracy with increased sampling. Despite encouragement only four participants attempted the Tru-Cut method.

Conclusions The session was popular and significantly improved trainee confidence. However, histological biopsy success rate and macroscopic correlation was poor. This is most likely related to difficulty in biopsying non-diseased pleura and possibly differences between macroscopic appearances of porcine and human pleura or inexperienced trainees' inability to recognise pleura macroscopically. Experience was low even in a high-incidence region, suggesting simulated pleural biopsy training may be a useful adjunct.

\section{REFERENCES}

1. Brown J et al. Abrams-Needle Pleural Biopsy Remains a Useful Investigation in Suspected Pleural Tuberculosis. Thorax 2012;67(Suppl 2):A113-A113.

2. Naicker TR et al. Validation of a novel resin-porcine thorax model for chest drain insertion training. Clinical medicine 2012;12:49-52.

\section{P75 PULMONARY PHYSIOGICAL TESTS: TRAINEES EXPERIENCE AND EXPOSURE}

${ }^{1} \mathrm{H}$ Lockman, ${ }^{2} \mathrm{NJ}$ Bell; ${ }^{1}$ Universiti Sains Islam Malaysia, Kuala Lumpur, Malaysia; ${ }^{2}$ Bristol Royal Infirmary, Bristol, United Kingdom

\subsection{6/thoraxjnl-2013-204457.225}

Introduction Knowledge of pulmonary function test (PFT) is essential for every respiratory physician. The level of training and exposure to PFT varies depending on the local facilities, supervisors and the respiratory trainees themselves. Moreover in recently cardio-pulmonary exercise testing (CPET) are becoming more widely available as well.

Objectives

1. To test the knowledge of UK respiratory physicians (trainees mainly) on PFT and CPET, identifying what is available locally.

2. The level of exposure to the tests themselves in terms of how to perform, the process involved and the equipment used/ available locally.

Methodology An electronic survey was distributed to the UK postgraduate deaneries for all the respiratory trainees and also to some respiratory physicians, thoracic surgeons and lung function physiologists. Feedback was collected anonymously over a period of 6 months (Dec 2012-May 2013). The questions ranged from simple spirometry, PFT, CPET and basic demographics.

Results A total of 160 responses were obtained from 16 deaneries out of 20 [ 1 from outside the UK - OOPE]. 83 (53\%) were respiratory specialist/specialty registrars and 61(39\%) consultants.

2 respondents had never seen spirometry performed, and 28 (18\%) have not seen a PFT performed. Only $1 / 4$ have done a PFT themselves. 70\% have seen CPET, 29\% have done CPET, $75 \%$ have a CPET service locally with cycle ergometer (66\%) being the most common method to exercise the patient. Respiratory physiologists and respiratory physicians are the ones mainly reporting CPET results with anaesthetist a distant third.

Summary Most trainees have been exposed to spirometry but based on this survey almost $20 \%$ have yet to see a PFT performed. Understanding the process of how to do a PFT and CPET, experiencing it personally could influence the number of PFT requests. This aspect of respiratory specialty training is still insufficient based on the feedback of respiratory trainees who answered this survey.

\section{P76 INVESTIGATING WOMEN'S EXPERIENCES OF ASTHMA CARE IN PREGNACY: A QUALITATIVE STUDY}

${ }^{1} \mathrm{C}$ Chamberlain, ${ }^{2} \mathrm{G}$ R Williamson, ${ }^{1} \mathrm{~B}$ Knight, ${ }^{1} \mathrm{M}$ Daley, ${ }^{1} \mathrm{DM}$ Halpin; ${ }^{1} R D \& E$ NHS Foundation Trust, Exeter, UK; ${ }^{2}$ Plymouth University, Plymouth, UK

\subsection{6/thoraxjnl-2013-204457.226}

Introduction and objectives Most asthmatic women have normal pregnancies and complications are infrequent when their asthma is controlled (BTS/SIGN 2012). Symptom control and medical treatment concern them, as does the impact of their illness and treatment on their unborn baby (Lim et al 2012). Few qualitative studies illustrate recently delivered asthmatic mothers' feelings about their care, support and medication during their pregnancy. 
The aim of the study was to investigate in a qualitative study the thoughts and feelings of women's experiences of asthma in pregnancy.

Methods NHS IRAS ethical approval and trust research governance were obtained; women gave written informed consent subject to the usual ethical guarantees. Twenty-two women with asthma and a pregnancy within two years were invited to participate. Seven women were interviewed when data saturation was achieved. Data collection took place between March 2012 and September 2012. Interviews were transcribed and analysed using a phenomenological 'Framework' Method involving familiarisation; identifying a thematic framework; indexing; charting; mapping; interpretation. Data were independently analysed by two researchers and consensus reached concerning themes.

Results

- Asthma and pregnancy

- Self-management including fears; lack of recognition of symptoms; poor knowledge of inhaled therapy

- Risk factors

- Anxieties including drugs, procedures, risks versus benefits

- General understanding of asthma

- Concealing symptoms

Pregnancy and post-natal experiences

- Impact of exacerbations on baby; breastfeeding benefits; changes to asthma, post-natal experience

Health professionals

- Lack of regular contact; midwife support; interaction with healthcare professionals, education

Conclusions These findings are globally relevant because maternal asthma is so prevalent. They illustrate participants' experiences of their asthma care and their views on its improvement. Similar to the international literature (Lim et al 2012), pregnant asthmatic women have concerns about their care and treatment, which might be alleviated by outreach, joint working between respiratory nurse specialists, midwives and GP practice nurses.

\section{REFERENCES}

1. British Thoracic Society (2012). British Guideline on the Management of Asthma. Available on-line at http://www.brit-thoracic.org.uk/Portals/0/Guidelines/AsthmaGuidelines/sign 101\%20Jan\%202012.pdf

2. Lim AS, Stewart K, Abramson MJ, Ryan K, George J (2012) Asthma during Pregnancy: The Experiences, Concerns and Views of Pregnant Women with Asthma. J Asthma. 49(5):474-9.

\section{P77 CREATION AND EVALUATION OF A 2D/3D MOLECULAR DATABASE FOR DRUGS USED TO TARGET THE RESPIRATORY SYSTEM}

SJ Cassar, C Shoemake, LM Azzopardi; University of Malta, Msida, Malta

10.1136/thoraxjn-2013-204457.227

Background Healthcare students and professionals could benefit greatly from using a holistic drug database as a reference point for clinical, physiochemical and structural information. ${ }^{1}$ The involvement of certain drug therapy in the management of a condition can be facilitated by using 2 dimensional and 3 dimensional renditions. ${ }^{2}$

Objectives To compile an electronic molecular database of drugs used in respiratory conditions to include constructions in two and three dimension, representations of drug protein interactions and to assess its utility by carrying out a randomised control study on a pharmacy student cohort.

Design A repository of drugs pertaining to 'respiratory system' section of the BNF 64 was created to include structural information using molecular modelling software ex. Symyx ${ }^{\circledR}, \mathrm{VMD}^{\circledR}$ and Sybyl ${ }^{\circledR}$, clinical and physiochemical information. The database was compiled and uploaded onto the University's website. A randomised control study using validated questionnaires was carried out on students reading for a degree in Bachelor of Science in Pharmaceutical Science. Their performance was assessed at baseline, after two weeks from using the database and four weeks after. Statistical results were generated using SPSS ${ }^{\circledR} 17$.

Results A total of, 46 2D structures and interactive 3D formats, and 21interactive representations of the 7PDB entries identified were created. A positive trend in student knowledge on drugs used in respiratory conditions was identified both immediately (post)and after a period of time (delayed), with students performing better after being exposed to the database during the intervention lecture as can be seen in Table 1. A significant improvement in the final marks was attained in the experimental group with respect to the control group for the first, second, third and fourth year undergraduate pharmacy students.

Conclusions Student understanding and knowledge is enhanced when teaching practices take on an innovative approach. In fact, $86 \%$ of the students deemed the electronic database to be a relevant reference point of information during the undergraduate course.

\section{P78 IMPACT OF A PHARMACIST-LED ASTHMA AND COPD REVIEWS IN GENERAL PRACTICE}

${ }^{1} \mathrm{H}$ Khachi, ${ }^{2} \mathrm{P}$ Karikari; ${ }^{1}$ Barts Health NHS Trust, London, UK; ${ }^{2}$ North East London Commissioning Support Unit - City \& Hackney, London, UK

10.1136/thoraxjnl-2013-204457.228

Introduction Asthma and COPD account for a significant burden of disease in the UK. Despite comprehensive guidelines, over a 1000 people continue to die from asthma each year and COPD accounts for a leading cause of emergency admissions in the UK.

A joint initiative between a tertiary centre for respiratory disease and commissioning support unit (CSU), asthma and COPD reviews were undertaken by a specialist respiratory pharmacist in GP practices. This study assesses the impact of these reviews.

Aims and Objectives This study aims to assess the impact of the specialist asthma and COPD reviews in accordance with national guidelines and standards of care.

Methods The study was carried out during eight clinical sessions over a two month period, across three GP surgeries. The reviews included the following assessments:

- Quality of life: ACT and CAT scores

- Inhaler technique

- Assessing adherence to maintenance inhalers based on GP prescription records

- Assessing if patients were on the correct therapy in accordance with their diagnosis, symptoms and severity of disease

Patients were identified for review based use of high dose inhaled corticosteroid and bronchodilator preparations (ICS/ LABA) and/or frequency of A\&E and hospital admissions.

Results During the eight clinical sessions, 84 patients with asthma or COPD were reviewed ( $42.5 \%$ male). $63 \%$ of patients 